\title{
Resection of a large rectal polyp with the simul- taneous combination of snare polypectomy and full-thickness resection device resection
}
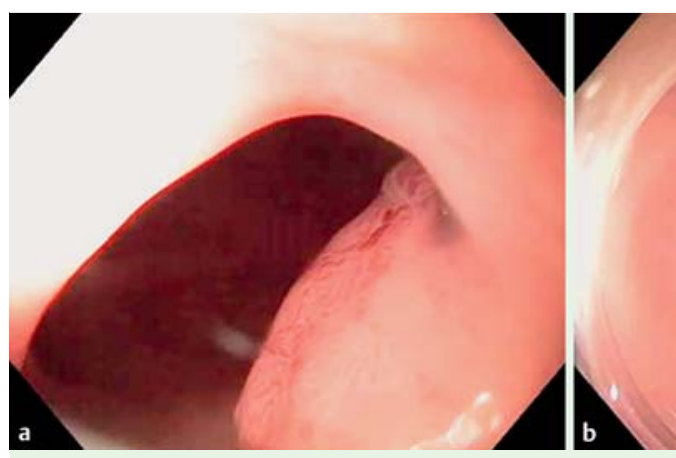

Fig. 1 Flexible endoscopy in a 59-year old woman referred for further treatment after incomplete polypectomy of a large rectal polyp, described as a tubulovillous adenoma harboring high grade dysplasia. a With a standard colonoscope, only part of the polyp remnant is visible. $\mathbf{b}$ Visualization of the true extent of the polyp remnant is improved with the mounted distance cap.
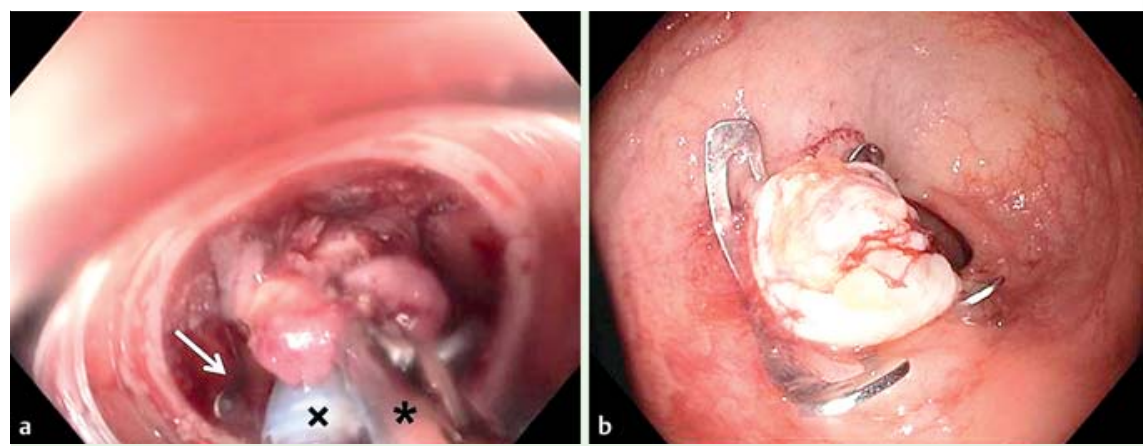

Fig. 3 Resection with the full-thickness resection device (FTRD). a The polypectomy site with visible small remnants of the polyp is pulled into the FTRD cap with the grasper (asterisk) included in the package. The polypectomy snare is preloaded on the FTRD cap (cross), and an over-the-scope clip (OTSC; arrow) is also loaded on the FTRD cap. b Full-thickness resection site with the OTSC in situ.

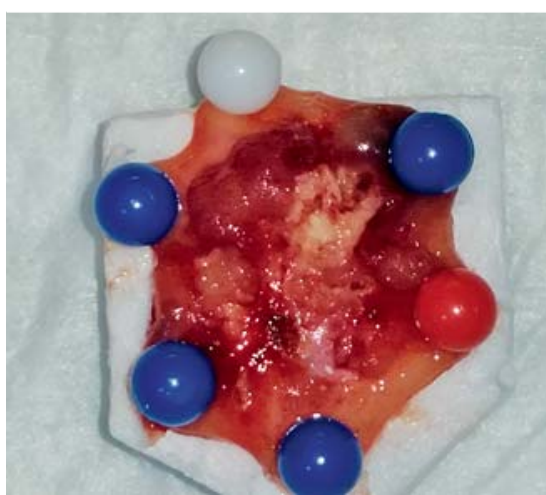

Fig. 4 The full-thickness resection specimen includes the R2 margin of the snare polypectomy. Based on the histological examination result, R0 resection was achieved.

A new device for full-thickness colorectal resection, the so-called full-thickness resection device (FTRD; Ovesco Endoscopy, Tübingen, Germany) has been available

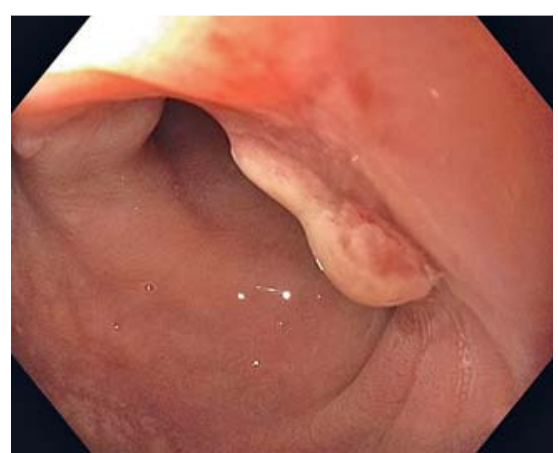

Fig. 5 Scar at 8 weeks after resection with the full-thickness resection device (FTRD). The clip has detached spontaneously. There is some granulation tissue in the center of the scar, typically seen early after FTRD resection.

since November of 2014. It is the first commercial system based on the overthe-scope clip (OTSC) principle. The FTRD consists of a modified 14t OTSC mounted

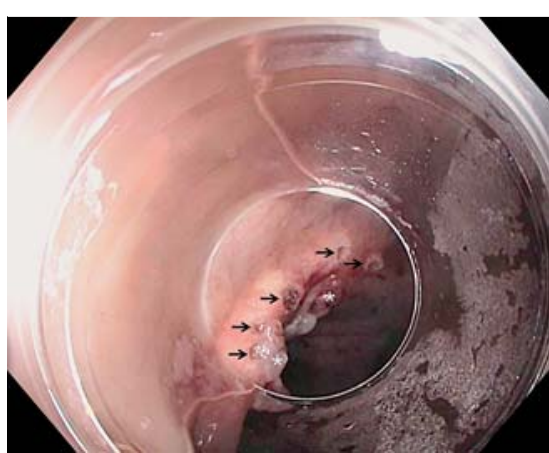

Fig. 2 Endoscopic view of the polyp base after snare resection. The lateral margins of small polyp remnants (asterisks) have been marked with the coagulation probe (arrows) included in the package of the full-thickness resection device.

on a transparent cap, which has a monofilament polypectomy snare preloaded on its tip. The FTRD can be mounted over a standard colonoscope [1]. Because the volume of the transparent FTRD cap is greater than that of the OTSC cap $\left(3 \mathrm{~cm}^{3}\right.$ vs. $0.9 \mathrm{~cm}^{3}$ ), full-thickness specimens with a median resection surface of $5 \mathrm{~cm}^{2}$ (range $1.6 \mathrm{~cm}^{2}-12.9 \mathrm{~cm}^{2}$ ) can be obtained [2].

A 59-year old patient was referred for further treatment after incomplete polypectomy of a large rectal polyp. Histologically, a tubulovillous adenoma harboring high grade dysplasia was described. The index endoscopic procedure demonstrated a villous polyp in the rectosigmoid curve that was difficult to visualize. With a mounted transparent distance cap (MAJ663; Olympus, Tokyo, Japan), better visualization was possible, and a large, broadbased villous polyp remnant about $3 \mathrm{~cm}$ in diameter was seen ( $\bullet$ Fig. 1).

After the patient had provided informed consent, polypectomy of the polyp remnant was performed close to its base with a standard snare (FlexSnare; Medwork, Höchstadt/Aisch, Germany; Fig.2). Then, the FTRD was mounted on a colonoscope (CF-H180AI, Olympus), and a fullthickness resection of the entire polypectomy site was carried out uneventfully $(\bullet$ Fig.3). The snare polypectomy remnant and the FTRD specimen measured $3 \times 2 \times 1 \mathrm{~cm}$ and $3.1 \times 2.5 \times 0.8 \mathrm{~cm}$, respec- 


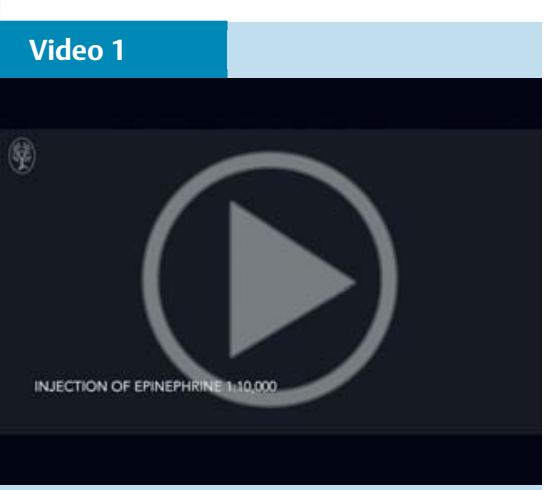

Resection of a large rectal polyp with the simultaneous combination of snare polypectomy and full-thickness resection device resection.

tively ( $\bullet$ Fig.4). The entire procedure is presented in $\bullet$ Video 1.

Histological examination confirmed fullthickness resection. The polyp remnant contained low grade dysplasia, and the FTRD remnant contained parts of the tubulovillous adenoma with negative resection margins. At follow-up 8 weeks later, the OTSC had detached spontaneously. A normal scar with some granulation tissue (typical after FTRD resection) was seen (๑ Fig. 5).

To our knowledge, this is the first report of simultaneous snare polypectomy and FTRD resection. The volume of the polyp was too large for FTRD resection alone, and it would not have fit into the FTRD cap.After the snare resection, clearly no $\mathrm{R} 0$ resection had been obtained. We decided on the simultaneous snare polypectomy and FTRD resection in order to avoid scarring and the development of tissue fibrosis. For successful FTRD resections comparable to OTSC treatment [3], tissue mobilization into the FTRD cap is crucial. At a later time, this might be complicated or even prevented by the healing process after the resection of a large polyp [1 - 3]. In conclusion, if incomplete snare polypectomy can be expected, additional resection of the polyp base remnant with the FTRD in the same session may be reasonable. Informed consent should be obtained before the procedure, and the necessary materials must be available.

Endoscopy_UCTN_Code_TTT_1AQ_2AD

\section{Competing interests: None}

\section{Andreas Fischer, Richard F. Knoop, Christine Walker, Robert Thimme, Hans-Jürgen Richter-Schrag}

University Hospital Freiburg, Interdisciplinary Gastrointestinal Endoscopy, Department of Medicine II, Freiburg,

Germany

\section{References}

1 Schmidt A, Bauerfeind P, Gubler C et al. Endoscopic full-thickness resection in the colorectum with a novel over-the-scope device: first experience. Endoscopy 2015; 47: 719 725

2 Richter-Schrag HJ, Walker C, Thimme R et al. Full thickness resection device (FTRD): experience and outcome for benign neoplasms of the rectum and colon [in German]. Chirurg 2015 Oct 5. [Epub ahead of print]

3 Haito-Chavez Y, Law JK, Kratt T et al. International multicenter experience with an overthe-scope clipping device for endoscopic management of GI defects (with video). Gastrointest Endosc 2014; 80: 610-622

\section{Bibliography}

Dol http://dx.doi.org/

10.1055/s-0035-1569658

Endoscopy 2015; 47: E607-E608

(c) Georg Thieme Verlag KG

Stuttgart · New York

ISSN 0013-726X

\section{Corresponding author}

\section{Andreas Fischer, MD}

Interdisciplinary Gastrointestinal Endoscopy

Hugstetterstr. 55

79106 Freiburg

Germany

Fax: +49-761-27025411

andreas.fischer@uniklinik-freiburg.de 